ESJ Humanities

\title{
L'Écriture du Dieu : Libération de la pensée humaine des banalités telluriques
}

\author{
Majida Sayegh
}

Faculté des Lettres et des Sciences Humaines (Section 5)

Département de Langue et Littérature Françaises, Université Libanaise

Doi:10.19044/esj.2021.v17n33p43

Submitted: 03 August 2021

Accepted: 23 August 2021

Published: 30 September 2021
Copyright 2021 Author(s)

Under Creative Commons BY-NC-ND

4.0 OPEN ACCESS

Cite As:

Sayegh M. (2021). L'Écriture du Dieu : Libération de la pensée humaine des banalités telluriques. European Scientific Journal, ESJ, 17 (33), 43.

https://doi.org/10.19044/esj.2021.v17n33p43

\section{Résumé}

Dans "L'Écriture du Dieu", Borges nous présente Tzinacan, le dernier prêtre de la pyramide de Qaholom, torturé et emprisonné. Armé de sa persévérance contemplative, il lutte pour se délivrer des entraves matérielles de sa prison et de son corps martyrisé par d'interminables années de captivité. Afin d'atteindre un affranchissement corporel et moral définitif, il entreprend la quête d'une formule fatidique de quatorze mots dont chacun contient toute l'entropie de l'univers. Mais avant d'y parvenir, il doit passer par des épreuves liées à toutes sortes de labyrinthes effrayants : depuis ses propres souvenirs, ses réflexions sur la vie et sur le langage, jusqu' aux rêves du sable, étouffants, emboités et infinis. Ces cauchemars le poussent à changer de stratégie dans sa recherche de l'énigmatique formule céleste qui lui donnerait tous les pouvoirs sur les autres humains à condition de la prononcer, mais la survenue de l'union avec le divin lui fit oublier sa propre identité ainsi que toutes ses préoccupations telluriques. Submergé par la béatitude, il s'enfonce définitivement dans l'oubli éternel pour partager avec tous les êtres un monde anonyme et infini.

Mots-clés: Écriture du Dieu, pensée tellurique, prison, souvenir, formule, infini, éternité, labyrinthe, apothéose 


\title{
The Writing of God: The Liberation of Human Thought from Telluric Banalities
}

\author{
Majida Sayegh \\ Faculty of Letters and Human Sciences (Section 5) \\ Department of French Language and Literature, Lebanese University
}

\begin{abstract}
In "The Writing of God", Borges introduces us to Tzinacán who is the last priest of the pyramid of Qaholom. Tortured and imprisoned, yet armed with his contemplative perseverance, he struggles to free himself from the material shackles of his prison and his body was martyred by endless years of confinement. In order to reach a definitive physical and moral emancipation, he embarks on a quest for a fateful formula of fourteen words, each of which contains all the entropy of the universe. Before succeeding, he must go through numerous trials in terrifying labyrinths. This ranges from his own memories, his reflections on life and on language, to the dreams of sand, stifling, interlocking, and infinite. These nightmares pushed him to change his strategy in his quest for the enigmatic celestial formula that would give him ultimate power over other humans, so long as he pronounces it. His union with the divine, however, made him forget his own identity as well as all his telluric worries. Overcome by bliss, he sinks into eternal oblivion thus sharing with all beings an anonymous and infinite world.
\end{abstract}

Keywords: Writing of God, telluric thought, prison, memories, formula, infinite, eternal, labyrinth, apotheosis

\section{Introduction}

Lire un texte de Jorge Luis Borges (1899-1986), c'est plonger dans le monde d'une littérature de dimension philosophique et contemplative qui célèbre la vérité cosmique libre et illimitée. Cet écrivain sensible au hasard et amoureux des mathématiques choisit ses thèmes d'après ses voyages fictifs qui transportent le lecteur au-delà des démarcations physiques et culturelles pour toucher l'essence de l'existence humaine.

Il est sûr qu'il existe une sorte d'intimité entre Borges (1953), l'auteur du recueil "L'Aleph", et l'aléatoire comme le dit Brotherston (2011). Ce recueil signifie une totalité vécue par les sens et l'esprit, une épiphanie de tout l'univers et de tous les temps concentrés en un moment, une vision cosmique qui illustre la tradition philosophique de l'Amérique revêtant une dimension universelle. Les héros borgésiens vivent souvent d'intenses aventures de la 
mémoire infaillible de toute l'humanité. Submergés par le fantastique, leur imagination est en perpétuel va-et-vient entre les trois instants fondamentaux du temps : le passé, le présent et le futur.

Borges débute "L’Écriture du Dieu" par « La prison est profonde (147) » afin de souligner l'intensité des épreuves de Tzinacan dans son cachot. Ce souterrain en pierre ayant la forme d'une « demi-sphère presque parfaite (147) » que cache-t-il comme énigmes ? Comment se manifeste la souffrance des prisonniers, d'un côté, Tzinacan, et de l'autre, " un jaguar qui mesure à pas égaux et invisibles le temps et l'espace de sa cellule (147) ». Pourquoi ce partage inattendu de ce lieu exigu et contraignant entre le dernier prêtre du peuple maya et cet animal féroce ? Entre ces deux créatures captives, apparaît furtivement un geôlier silencieux qui ouvre la trappe pour leur descendre un morceau de viande et une cruche d'eau chaque jour à midi. Terrassé et broyé par de très longues années de séquestration morbide, l'objectif ultime de Tzinacan était de se libérer à tout prix. Le pelage jaune du jaguar, symbole religieux chez les Mayas, semble renfermer un secret divin transmis à travers les générations.

En effet, Tzinacan est « le mage de la pyramide de Qaholome, qui fut incendiée par Pedro de Alvarado ${ }^{1}$ (147)». Il porte en lui toutes les souffrances du peuple maya. Dans ce temps immémorial, les fameux textes de Chilam Balam constituent un des plus importants témoignages de la littérature maya yucatèque où apparaissent divers dieux qui interviennent dans la conduite des mondes supérieur et inférieur ${ }^{2}$. Imbu de cette culture tournée vers une transcendance bienveillante, le mage emprisonné, torturé et humilié cherche une issue salvatrice de ses supplices.

Dans cette recherche, une méthode d'investigation a été utilisée pour accompagner Tzinacan dans sa quête. En effet, le volet pratique de cette

${ }^{1}$ Pedro de Alvarado (1485-1541): Un conquistador espagnol qui a participé à la conquête de l'empire aztèque sous les ordres d'Hernando Cortes. Alvarado est également connu pour la cruauté de son traitement des populations autochtones, et les meurtres de masse commis pour assujettir des peuples indigènes du Mexique. Les conquérants n'ont pas seulement détruit hommes, femmes et enfants mais une civilisation humaine d'une richesse inestimable. En 7 Juillet, 1520 il y a eu la Bataille d'Otumba pendant laquelle les féroces guerriers du jaguar et de l'aigle, les hommes de conquistador ont arraché une victoire contre les aztèques. Voir Zinn (2009),

https://bruxelles-panthere.thefreecat.org/, https://www.matierevolution.fr/spip.php, https://jugurtha.noblogs.org/files/2018/05/HowardZinn-HistoirepopulairedesEtats-Unis.pdf ${ }^{2}$ Ces livres, qui représentent l'un des plus importants témoignages de la vie des peuples autochtones, tiennent un registre de leur civilisation : histoires, mythes, traditions, dieux, folklores, médecine et astronomie, etc. Chilam (ou Chilan) signifie « l'interprète des dieux » et Balam signifie "jaguar », mais c'est aussi un nom de famille commun dans le Yucatan, situé au sud du Mexique, de sorte que Chilam Balam pourrait bien être traduit comme le Prophète Balam (Shroukh, 2020) 
recherche fait appel à une sorte d'analyse d'investigation pour effectuer une enquête dans les constituants du texte. Le comportement imaginatif de Tzinacan l'a rendu comme un enquêteur de la vérité de l'existence cosmique. Des voyages seront effectués dans des labyrinthes étranges et mystérieux inspirés par la vie de la nature : des réseaux de tigres et des rêves emboités de sable avec leurs différentes interprétations. Des parcours entre le fini et l'infini seront établis en se servant avec précaution de certaines notions scientifiques adoptées par Borges et citées comme des instruments analytiques scientifiques par les chercheurs.

Cet article est divisé en deux grandes sections. La première est destinée à analyser les labyrinthes, matériels et immatériels vécus par le mage. La deuxième consiste à explorer l'effort de Tzinacan pour s'affranchir de tous ces labyrinthes pour se confondre avec la divinité et l'univers.

\section{Les labyrinthes vécus par Tzinacan}

Dans son avertissement de "L'Aleph", Caillois évoque que Tzinacan, un mage aztèque «prisonnier dans la pire oubliette, parvient à lire L'Écriture du Dieu et à pénétrer l'énigme de l'univers ». D'abord, la prison profonde en pierre est décrite nettement : elle a la forme «d'une demi-sphère presque parfaite (147) ». Pourquoi cette forme est-elle imaginée par Borges ? En effet, en mathématiques, la sphère est la forme parfaite dans le monde réel de trois dimensions. Elle est omniprésente dans les objets infiniment grands ou même infinitésimaux : les étoiles, les perles, les gouttes d'eau (privées de gravité) et même les atomes ainsi que leurs constituants de protons et d'électrons. Les astres et les planètes voguent selon une orbite dans l'univers dont le vide constitue $90 \%$ de son volume. Pour cela, une sphère représente le symbole de mouvement (Lavarde, 1999).

Dans ses ouvrages "Histoire de l'éternité", et "Le Temps circulaire", Borges perçoit le temps comme un cercle où chaque point se confond avec luimême après un tour complet, et par conséquent cela amène l'individu à repasser une infinité de fois par le même point. Il précise aussi que cette concordance entre les points de départ et d'arrivée rend l'histoire universelle comme étant l'histoire d'un seul être humain, et chaque instant représente toute une vie (Borges, 1936, 1971). Dans son recueil de nouvelles «Le livre de sable », Borges (1975) justifie le choix de ce titre en disant «parce que ni ce livre ni le sable n'ont de commencement ni de fin (100) ». Il ajoute « Si l'espace est infini, nous sommes dans n'importe quel point de l'espace. Si le temps est infini, nous sommes dans n'importe quel point du temps (100)». En effet, la présence d'un cercle évoque plusieurs symboles $^{3}$ : «l'unité, l'harmonie, l'infini et le domaine de l'esprit. Il est à la fois début et 
fin, fini et infini, tout et rien ». Selon (Nicolas, 1999), le cercle rappelle « la quête sans fin, la limite asymptotique vers une droite ». Le cercle a aussi un sens égalitaire : la table ronde, par exemple, accorde l'égalité des membres qui sont rassemblés autour d'elle. Dans la mythologie aztèque, le cercle évoque l'Ouroboros: le dieu serpent à plumes des Aztèques, Quetzalcoalt, fréquemment représenté enroulé sur lui-même est le symbole de la PuissanceDivine qui n'a ni commencement ni fin. Selon les Incas, Huira Cocha, le dieu créateur et héros civilisateur (Mathieu-Colas, 2017) est l'Esprit de l'abîme ou de la mer, aussi l'Esprit incommensurable, son symbole était le soleil levant sortant des eaux ${ }^{4}$. Par ailleurs l'Ouroboros est à la fois autodestruction et renouvellement, anéantissement et vie.

Borges revêt sa description de la cellule de Tzinacan d'un aspect géométrique dont la circularité est entravée :

«La prison est profonde. Elle est en pierre. Sa forme est celle d'une demi-sphère presque parfaite ; le sol, qui est aussi en pierre, l'arrête un peu avant le plus grand cercle, ce qui accentue de quelque manière les sentiments d'oppression de l'espace. Un mur la coupe en son milieu. Il est très haut, mais n'atteint pas la partie supérieure de la coupole (147)».

Cette architecture pleine de barrières physiques lui causait une angoisse permanente. Le seul «instant de lumière (150)» était quand le geôlier ouvre la trappe à midi pour quelques instants. Assoiffé de la vue du jaguar, son compagnon de prison, le mage profite de ce bref temps pour entrevoir ses mouvements et ses taches noires. En effet, apprendre l'ordre et la disposition de ces taches exigeait de Tzinacan de longues années pour les fixer dans sa mémoire (150).

Des années ont passé et Tzinacan souffre seul dans son cachot, un lieu étroit et figé où les ténèbres se répètent en mouvement cyclique et interminable. En se rappelant à peine de sa jeunesse, le mage assiste à une double perte : sa force et sa civilisation. Affaibli dans son corps et incapable de marcher, son seul espoir était d'attendre un signe des dieux (148).

Tzinacan est condamné à chercher la chose dans son contraire : la liberté dans la prison, l'émancipation dans l'oppression, la lumière dans les ténèbres. Paradoxalement les labyrinthes semblent les seuls moyens pour entrevoir une solution. Les épreuves de Tzinacan n'auraient pas de sens sans ce passage obligatoire dans ses propres labyrinthes infernaux. Ils sont la concrétisation de ses efforts pour atteindre la lumière. Les sans-issues par nature seront le moyen d'accéder à la liberté dans la mesure où la descente aux ${ }^{4}$ Il semble que ce dieu n'est pas d'origine péruvienne mais il vient d'un pays où le soleil sort
des eaux où la mer est à l'orient (Girgois, 2015). 
enfers s'avère intérieure avant d'être extérieure. C'est ce qu'affirme Mottet (2010) en écrivant : "Borges s'égare délicieusement dans son labyrinthe, jusqu'à s'y dissoudre au profit des vérités universelles ».

A partir de cette nouvelle, essayons donc de trouver les profils de ces différents labyrinthes dans lesquels Tzinacan devait se perdre avant d'arriver à la lumière.

\section{Le labyrinthe de l'emprisonnement ténébreux}

Le premier des labyrinthes est attaché aux circonstances mêmes de son emprisonnement. Les ténèbres imposées à Tzinacan effacent l'écoulement du temps et le détachent de tous les repères spatio-temporels : « Un jour ou une nuit - entre mes jours et mes nuits, quelle différence y a-t-il ? (151)». Une telle déclaration indique l'écoulement excessif des jours inutiles à compter. La routine infernale des ténèbres est entrecoupée par l'ouverture furtive de la trappe. Ces supplices infinis sont l'incarnation typique du labyrinthe. En effet, la succession des heures était très similaire et pesante où chaque jour est une copie de ce qui le précédait et un présage de ce qui le suivait. Sachant que l'absence d'issue, la répétition et l'aspect identique et inchangeable des choses et des événements est le signe distinctif du labyrinthe. Rappelons l'architecture de la Cité surhumaine dans «L'Immortel » et l'aspect effrayant des Troglodytes qui représentent l'esprit labyrinthique par excellence ${ }^{5}$. Cette succession inexorable des jours et des actes mécaniques du geôlier contrastait profondément avec le sens de la vie humaine qui suppose une évolution et un aboutissement. C'est la suspension du temps réel et l'effacement des repères de l'espace pour maintenir une survivance morbide. Désarmé et enfermé dans son présent et incapable d'entrevoir un avenir, les pistes s'avèrent exclusivement du côté du temps d'avant la captivité, seul domaine où l'entité humaine était encore libre et vivante. Tzinacan veut puiser dans son histoire lumineuse et s'inspirer des idées de sa propre civilisation. Il s'accroche à sa mémoire comme seule ressource contre l'anéantissement et l'effacement. Ainsi la réminiscence apparaît comme un moyen de reconquérir l'énergie créatrice d'une force réelle. Mais sa mémoire se révèle un labyrinthe enchevêtré et ardu. Son combat vise à retrouver l'essence du temps qui n'aura aucune valeur si l'acte de souvenir devient impossible, c'est que confirment (Fontcuberta, Borges, \& Bleton, 2000) en mentionnant que Borges « a formulé que le souvenir est aussi prodigieux que la divination, car passé et avenir sont des parcours de temps, or le temps est pure illusion».

${ }^{5}$ «J'étais passé par un labyrinthe, mais la très nette Cité des Immortels me fit frémir d'épouvante et de dégoût [...] Un labyrinthe est une chose faite à dessein pour confondre les hommes ; son architecture, prodigue en symétries, est orientée à cette intention. Dans les palais que j'explorai imparfaitement, l'architecture était privée d'intention ». L'Immortel, P. 23. 
En effet, sans la faculté de la mémoire, Tzinacan se perdrait complétement dans l'obscurité de son cachot. Seule la mémoire revigore l'esprit du mage vu la dégradation de son corps : sa vie chargée d'expériences se réveille grâce à sa volonté de se retrouver pour chercher le secret transcendant qui pourrait l'aider à se dépasser. Devant cet objectif, il n'a pas eu recours à l'imagination, à la création nouvelle, mais à la remémoration car il était sûr de la valeur de son passé et de sa mission en tant que dernier prêtre de son peuple. Dans ce contexte va le philosophe français Henri Bergson (1896, 2011, p. 164) en distinguant entre image et souvenir : «Imaginer n'est pas se souvenir. Sans doute un souvenir, à mesure qu'il s'actualise, tend à vivre dans une image ; mais la réciproque n'est pas vraie, et l'image pure et simple ne me reportera au passé que si c'est en effet dans le passé que je suis allé la chercher, suivant ainsi le progrès continu qui l'a amenée de l'obscurité à la lumière ».

\section{Le labyrinthe de la mémoire}

Selon (Borges, 1969, p.157) « Nous sommes notre mémoire, nous sommes ce musée chimérique de formes inconstantes, cet ensemble de miroirs cassés ». Tzinacan va vivre les méandres de la mémoire qui multiplient les images et confondent la réalité et son reflet. Voyager dans sa propre mémoire c'est entreprendre un voyage magique, incertain et dynamique. Pour ce mage, essayer de se remémorer, ce n'est pas revisiter des vestiges inanimés, mais replonger dans une eau mouvante et revigorante. Cet acte volontaire est un cheminement intellectuel ciblé et non pas un abandon paresseux.

Pour Tzinacan, le retour au passé prend l'allure d'une quête et d'un itinéraire initiatique : au départ, il cherchait dans les souvenirs un refuge contre l'anéantissement et la dissolution, mais au fur et à mesure de son avancement rétrospectif, il allait vers un but encore plus défini symbolisé par la formule divine qui résume toutes les aspirations pour lui. C'est le Graal spirituel par excellence. En effet, à partir des connaissances élémentaires, Tzinacan tente d'accéder à d'autres plus exhaustives et parfaites qui contiennent le tout et répondent à toutes les questions.

Pour récupérer avec lucidité les images de son passé, Tzinacan a décidé de fouiller dans ses années antérieures et d'y revivre afin de les transformer en une étincelle qui allège l'obscurité imposée par ses geôliers. En rafraichissant sa mémoire, il a réussi à «peupler [son] temps (148) », et de cette manière il a pris "possession de tout ce qui [lui] appartenait (148)». Avant d'arriver à son précieux souvenir, il devait refaire le trajet de toutes ses connaissances. Mais un souvenir illumine soudain sa mémoire et verse dans son cœur l'espérance : une formule divine inscrite quelque part depuis le premier jour de la création et qui peut sauver tout : 
« Une nuit, je sentis que j'approchais d'un souvenir précieux. [...] Prévoyant qu'à la fin des temps se produiraient beaucoup de malheurs et de ruines, il écrivit [par référence à Dieu] le premier jour de la création une sentence magique capable de conjurer tous ces maux. Il l'écrivit de telle sorte qu'elle parvienne aux générations les plus éloignées et que le hasard ne puisse l'altérer (148-149)».

L'émergence de cette idée serait l'issue de ce labyrinthe de la mémoire qui devait déboucher sur un résultat salvateur.

La réponse à la question du présent semble venir du passé et non pas de la divination. C'est le souvenir de Tzinacan qui doit le secourir pour tracer son cheminement vers la liberté, l'universalité et l'éternité car "sur toute l'étendue de la terre, il existe des formes antiques, des formes incorruptibles et éternelles. N'importe laquelle d'entre elles pouvait être le symbole cherché (149) ». La difficulté à trouver ce Graal ne réside pas dans son unicité mais dans son multiplicité à l'infini : il est partout et nulle part. Le défi est dans la capacité de le saisir et de le comprendre. Ainsi, «trouver » devient synonyme de « comprendre » et de «s'approprier » chez Borges, d'où l'aboutissement initiatique par excellence. C'est pourquoi la recherche de cette formule libératrice se révèle un vrai calvaire qui oblige celui qui avait su rester silencieux sous la torture de ses geôliers de crier aux murs car il était incapable de déchiffrer ce qui est tracé sur la peau du jaguar qui aurait renfermé la formule sacrée : «Quelques-unes figuraient des points, d'autres formaient des raies transversales sur la face intérieure des pattes; d'autres, annulaires, se répétaient. Peut-être était-ce un même son ou un même mot. Beaucoup avaient des bords rouges (150) ». Ainsi, le mage cherchait une issue au labyrinthe de sa pensée. Cette énigme à résoudre le tourmentait « moins que l'énigme générique que constitue une sentence écrite par un dieu (151)». Mais Tzinacan s'interroge au fond de soi-même sur l'essence même de cette formule divine : "Quelle sorte de sentence, me demandais-je, devait formuler une intelligence absolue ? Je réfléchis que, même dans les langages humains, il n'y a pas de proposition qui ne suppose pas l'univers entier (151) ». Et c'est ce qui l'a rendu si anéanti et désespéré en se trouvant coincé dans un dédale non matérialisé qui était au fond de nature philosophique. La descente aux enfers physique et matérielle représentée par l'emprisonnement s'estompe devant l'acuité des tortures intellectuelles liées à sa recherche.

\section{Le labyrinthe chaotique de la vie naturelle}

Chaque pensée de Tzinacan est truffée d'enchevêtrements inextricables telle sa recherche constante de la loi qui gère la manifestation de la vie sur terre. Dans ses réflexions profondes, il imagine un labyrinthe dessiné 
par les lois de la nature et des créatures qui se déplacent sur terre et en mer. Sa vision holistique dépasse les limites de l'espace et du temps. Tzinacan enrichi par une vision globalisante selon un "œil circulaire" qui exprime son refus d'être emprisonné par la matérialité des choses. Ce regard holiste lui permettait d'embrasser la totalité de l'existence dans toutes ses manifestations à la fois apparentes et cachées. Le jaguar, compagnon de captivité, va produire tout un réseau d'images liées à la complexité de la vie de la nature. La grandeur des choses et leurs mystères se trouveraient ainsi du côté du visible offert généreusement par le cosmos et c'est à l'homme d'en déchiffrer les symboles et les interpréter. A cet effet, le labyrinthe devient un constituant naturel de la manifestation de la vie cosmique. Une sorte d'auto-défense de la vie contre la mort. Ainsi le labyrinthe sort de la catégorie de la destruction pour rejoindre celle de la protection et se révèle presque une nécessité. Tel est le cas provoqué par l'action des tigres. Pour Tzinacan, un réseau de tigres révèle un «brûlant labyrinthe (150) ». Mais «dire "le tigre", c'est dire les tigres qui l'engendrèrent (151) ». Ces tigres comme d'autres prédateurs répandent «l'horreur dans les prés et les troupeaux (150)».

Imaginons les pas de cet habile chasseur, un pas après l'autre, une sorte de "marche silencieuse" qui reflète un excès de confiance, d'assurance et de détermination pour attraper sa proie. Un réseau de tigres attaquant un troupeau de cerfs dans une vaste plaine produirait une scène de déplacements chaotiques par excellence : chaque cerf court dans tous les sens, de haut en bas, de gauche à droite, dans des mouvements ondulatoires, dans un seul objectif de se sauver d'une mort hallucinante. En accélérant sa vitesse devant le tigre, le cerf change brutalement sa direction, c'est comme s'il rencontrait brusquement une voie sans issue en faisant l'aller-retour avec toute sa force, une sorte de répétitions en traçant un labyrinthe non matérialisé, cauchemardesque et très complexe. Ainsi, l'essence du labyrinthe réside dans l'aspect chaotique des mouvements en changement perpétuel, ce qui provoque égarement certain. Tout est soupçonné porter la formule divine. Même l'accouplement et l'engendrement de ces jaguars n'auraient pas pour but que la préservation et la transmission de la fameuse écriture. Effectivement, le cheminement de la recherche de Tzinacan est graduel et va en commençant par toute chose existante et qui pourrait, selon sa définition, être porteuse de cette écriture divine. Cependant, l'Écriture du Dieu ne ressemble pas aux écritures des hommes qui nécessitent des signes linguistiques et des moyens techniques pour s'inscrire. L'homme écrit pour enregistrer ses idées et pour qu'il soit capable de les revoir quand il en a besoin ou de les transmettre aux autres. Sans écriture, l'histoire de l'homme resterait dans les ténèbres éternelles de l'inconnu. Mais Tzinacan est conscient des "pauvres mots ambitieux des hommes (151) » qui ne peuvent en aucun cas exprimer l'immensité de l'univers et sa totalité. Il se rend compte que l'écriture du Dieu 
ne peut ressembler à aucune autre écriture car elle exprime l'universel et l'éternel qui dépasse tous les repères. Pour y accéder il faut briser les labyrinthes spatio-temporels pour reconstituer l'écoulement de l'histoire non seulement de l'homme mais aussi l'histoire du cosmos lui-même.

\section{Labyrinthe : le rêve de sable}

Le rêve de Tzinacan est un moyen de délivrance de cette situation pénible et non pas une échappatoire. Il est aussi précieux que la réalité. En effet, le combat de Tzinacan trouve son apogée dans ses rêves. Les labyrinthes de la mémoire et des interrogations philosophiques sur la nature de la formule supposée de Dieu aboutissent à la vision cauchemardesque des rêves de sable. C'est l'allégorie des angoisses terrestres dont le comble va se concrétiser dans un grain de sable. Dans sa symbolique tellurique et sa consistance matérielle, le sable infini et solide représente la rigidité de la terre : à l'encontre de l'eau et du feu, il est par excellence l'empêchement de toute mobilité et de toute délivrance. Chaque grain de sable est un monde matériel et la prolifération de celui-ci crée l'inextricable. En réalité, la multiplication de l'identique est la première caractéristique du labyrinthe. D'où l'excès de ces rêves de sable emboîtés qui symbolisent l'apogée des supplices intellectuels de Tzinacan. Ce sont l'emblème des dédales telluriques qui ont envahi l'esprit du prisonnier tourmenté par des questions sans réponses. Le réveil impossible dont il est question serait l'aboutissement clair et net de ses interrogations sans fin. Cette confusion mentale signifie l'emprise excessive et atroce de la réalité matérielle.

Il rêvait que sur le sol de la prison, « il y avait un grain de sable ${ }^{6}\left(R_{1}\right)$. [...], deux grains de sable $\left(R_{2}\right)$ [...], les grains de sable étaient trois $\left(R_{3}\right)$. Ils se multiplièrent ainsi jusqu'à emplir la prison (151) », puis une chaine infinie de rêves emboitées (rêve du rêve) $\left(R_{1} \rightarrow R_{2} \rightarrow R_{3} \rightarrow \cdots \rightarrow R_{n} \ldots\right)$ se produit, une sorte d'un espace infini formé par des entiers naturels caractérisant le nombre de grains de sable dans chaque rêve. C'est du genre des poupées russes, " c'est comme si, en représentant une réalité irreprésentable dans le rêvé du rêve » comme dit (Civitarese, 2015). Le chiffre trois est utilisé ici comme le dernier chiffre qui informe sur le fini. De plus une information importante pourrait être déduite du volume infini de sable: imaginons le nombre infini des couloirs formés grâce aux interstices résultant des disposions des grains de sable, les uns contre les autres. Dans ce monde

${ }^{6}$ D'après Éric Chaumillon (2016), la taille d'un grain de sable est comprise entre 63 microns et $2 \mathrm{~mm}$ et d'un volume $1 \mathrm{~mm} 3$ environ. À l'échelle planétaire, le sable représente presque $1 / 3$ du total des sédiments et semble inépuisable car il est estimé à $12 \times 1016$ tonnes. Le nombre de grains de sable sur la planète serait ainsi équivalent au nombre d'étoiles dans l'univers. 
chaotique, une infinité de labyrinthes fantastiques se produisent et le fait d'y penser provoque un vertige.La prison est devenue un « hémisphère de sable » en faisant allusion à la forme de prison. Quelqu'un lui dit :

"Tu ne t'es pas réveillé à la veille, mais à un songe antérieur. Ce rêve est à l'intérieur d'un autre, et ainsi de suite à l'infini, qui est le nombre des grains de sable. Le chemin que tu devras rebrousser est interminable ; tu mourras avant de t'être réveillé réellement (152)».

Quel labyrinthe affreux fait errer Tzinacan dans ses rêves encastrés ? Un hémisphère de sable le presse sans pitié, un microcosme de souffrance l'entoure pour anéantir le dernier souffle vital. En effet, il s'agit de labyrinthes interminables dont les volumes augmentent d'un rêve à l'autre, et par conséquent, le cumul illimité des petits ou infiniment petits conduits à l'infiniment grand, donc parler de volume sera plus signifiant que de nombre. Ces rêves monstrueusement enchâssés sont l'emblème de l'idée emprisonnée à son tour à l'image du rêveur. Comme lui elle n'arrive plus à se libérer de la répétition insensée des contraintes spatio-temporelles. Un clin d'œil au prisonnier d'enfreindre les limites dans sa pensée pour parvenir à la certitude en dépassant tout repère réducteur de la liberté. Tzinacan dit: "le sable m'étouffait (152) », n'est-ce pas une allusion à la matière qui s'incruste dans la pensée en étouffant les rêves et les rêveurs et dont il faut se débarrasser pour atteindre l'immatériel, l'infini et l'éternel ?

\section{La sortie du labyrinthe immatériel : Fruit de patience et de clairvoyance}

Nourri par l'amour du divin, Tzinacan a pris l'initiative de raviver sa mémoire chargée de sagesse et d'espoir pour insuffler de la vie dans son existence ténébreuse de prisonnier. Avec sa sagesse fermement ancrée au plus profond de sa propre personnalité, il a pu résister à l'incapacité physique de son corps vieillissant. Les ailes de sa mémoire et de son imagination le transportent là où il a envie d'être partout dans l'existence. Avec cet effort mnésique et cette détermination invincible d'atteindre son but sacré qui consiste à retrouver la formule divine salvatrice, Tzinacan a récupéré la force de sa mémoire pour se connecter à des images et des connaissances que les années d'emprisonnement ont brouillées.

Sa recherche soupçonne tout comme porteur de cette transcription magique : tout objet et toute créature terrestre pouvait la porter quelle que soit sa nature. Même le firmament peut apporter des informations à son sujet. Mais ce qui le perturbe et l'attriste davantage, c'est les changements et les mutations qui peuvent toucher tout et fausser toute recherche. Après tant de suppositions et d'investigations lointaines, il revient à lui-même et s'approche de plus en 
plus du chemin intérieur qui pourrait le mener à l'objet de sa quête et revient au lieu même de sa prison comme un espace porteur de connaissance. Une façon de s'interroger sur le sens véritable de cet emprisonnement même. Comme s'il voulait donner sens à cette situation atroce dans laquelle il existe. La formule parfaite semble comme incrustée dans la personnalité même du prisonnier :

«Peut-être la formule était-elle écrite sur mon visage et j'étais moi-même le but de ma recherche. À ce moment, je me souvins que le jaguar était un des attributs du dieu (150)».

Comme il est constaté dans cette évocation, le jaguar représente un attribut de dieu $^{7}$, il désigne aussi un prophète comme mentionne Shroukh (2020) «Balam était surtout le nom d'un célèbre prophète maya [...], qui aurait vécu [...], peu avant l'arrivée des Espagnols. La renommée de ce prophète-jaguar tient au fait qu'il aurait prédit la venue d'étrangers porteurs d'une religion nouvelle ».

Ainsi, le fait de trancher que le secret de la formule divine se trouve dans les taches du jaguar, Tzinacan fixe un objectif nouveau: pouvoir déchiffrer ce message divin au fur et à mesure que la lumière de midi le permet pour quelques instants. Et encore une fois, c'est le travail de la mémoire qui va opérer pendant des années et des années. Ainsi il se trouve confronté à ce mystère doublement pénible :

« Je ne dirai pas mes fatigues et ma peine. Plus d'une fois, je criai aux murs qu'il était impossible de déchiffrer un pareil texte. Insensiblement, l'énigme concrète qui m'occupait me tourmenta moins que l'énigme générique que constitue une sentence écrite par un dieu. "Quelle sorte de sentence, me demandais-je, devait formuler une intelligence absolue $?(151) »$

Par cet intérêt porté à son voisin de cellule, Tzinacan donne sens à toute forme vivante qui partage avec lui les mêmes circonstances de survie. Il réussit ainsi à faire naître l'espoir du désespoir même. Il ouvre sa pensée à toutes les manifestations de vie ce qui élargit ce lieu d'enfermement atroce et

${ }^{7}$ Les souverains mayas considèrent le jaguar comme symbole du droit divin des rois. Le dieu Jaguar habitait le Monde d'en bas, la demeure des morts. Tous les matins, il devenait le dieu du Soleil et voyageait à travers le ciel, vers l'ouest, où il retombait dans le Monde d'en bas. Afin de faire perpétuer le cycle des jours et des nuits, les souverains accomplissaient des rites pour apaiser les dieux, dont dépendait le sort de l'humanité. Comme le dieu Jaguar, les rois mayas défiaient la mort en renaissant hors du Monde d'en bas, auquel l'humain moyen ne pouvait échapper. Cette citation est prise du site :

https://www.museedelhistoire.ca/cmc/exhibitions/civil/maya/mmj01fra.html\#. 
inhumain. Ce qui est spatialement exigu et fini devient infini et les quelques instants de lumière à midi deviennent ce qu'il y a de plus cher. La prison va devenir un lieu de découverte et d'expérience.

Malgré la lourde obscurité des labyrinthes de sable représentés par les rêves infinis et emboités, le destin de Tzinacan annonce implicitement l'existence d'une lumière qui se rapproche petit à petit pour s'établir dans sa pensée. N'ayant plus peur de cette obscurité et sa cruauté, il retourne à sa dure prison mais avec une nouvelle pensée créative et éclatante en disant :

« Je bénis son humidité, je bénis son tigre, je bénis le soupirail, je bénis mon vieux corps douloureux, je bénis ${ }^{8} 1$ 'obscurité de la pierre (152-153)».

En réalité, en sortant de son labyrinthe de sable, Tzinacan est retourné à sa dure prison préférée à ce rêve chaotique, monstrueux et étouffant. Son regard s'est porté sur son microcosme défini par cinq éléments proches de lui. En interagissant avec eux à tout moment, ils sont devenus une partie de son être moral. Grâce à son effort intellectuel persistant, il a pu arriver à saisir le sens de l'Écriture de Dieu et il s'y dissout.

Avec la conscience de Tzinacan de ce qui endure d'atrocité, il a pu minimiser l'impact des divers labyrinthes sur sa psyché, après les avoir attaqués avec sa pensée éclairée et sa mémoire ardente et assoiffée de liberté universelle. Tout cela l'a amené à se libérer après avoir été capable de comprendre ce que signifient les mots de Dieu : uniques et infinis, ils se distinguent par leur impartialité d'une part et leur infinité d'autre part. Dans ce sens, va Tzinacan en disant :

«Je réfléchis encore que, dans le langage d'un dieu, toute parole énoncerait cet enchaînement infini de faits, et non pas d'un mode implicite, mais explicite, et non pas une manière progressive, mais instantanée (151)».

En effet, la bénédiction du mage pour les cinq éléments est d'une extrême importance: il a commencé par bénir son entourage direct victime et torturé comme lui. C'était sa victoire sur ses bourreaux, sur sa vieille pensée accablée par un raisonnement tellurique géré par les contraintes d'espace et de temps :

«Alors arriva ce [qu'il ne peut] oublier ni communiquer. Il arriva [son] union avec la divinité, avec l'univers ([Il ne sait pas] si ces deux mots diffèrent) (153)».

\footnotetext{
${ }^{8}$ Il répète cinq fois "je bénis", en signalant que pour des alchimistes, le chiffre cinq est un nombre fétiche comme affirme Nicolas (1999), en numérologie, il symbolise « la mobilité, la liberté, le plaisir, l'affection et le dynamisme ».
} 
Une déclaration révélatrice qui consiste à se sauver de tout type de labyrinthes en rentrant dans l'espace divin élu comme demeure éternelle. Borges associe la divinité à l'univers, et par conséquent, pour pouvoir définir l'Écriture de Dieu, il a fallu réussir à déchiffrer l'Écriture de l'univers. Tzinacan a pris conscience que l'univers est le vrai foyer de toute créature et il a compris profondément que l'Écriture du Dieu est cette bibliothèque cosmique infinie. Sortir de son propre labyrinthe, c'est entrer dans l'univers, ce labyrinthe magique, et chaque homme peut y trouver une issue qui informe sur toutes les autres issues de nombre infini. Il dispose ainsi de son propre fil d'Ariane qui lui permettrait de se sauver des langages réducteurs pour pouvoir entrer dans la véritable langue universelle. Et comme le dit Borges dans sa nouvelle L'Aleph "La vérité ne pénètre pas dans un esprit rétif (204)», l'effort intellectuel déployé par Tzinacan est proportionnel avec son désir de liberté. Si certains voient Dieu dans une fleur ou une épine, et d'autres le voient dans un éclat, alors lui, il le voit dans la roue de l'univers, une roue faite d'eau et de feu. C'est arrivé juste au moment de son union avec le divin. L'affranchissement presque cosmique du mage se contraste superbement avec les murs aveugles de son prison. Tzinacan voit la Roue des mystères, des secrets, des destins :

«J'ai vu une Roue très haute qui n'était pas devant mes yeux, ni derrière moi ni à mes côtés, mais partout à la fois. Cette Roue était faite d'eau et aussi de feu et elle était, bien qu'on en distinguât le bord, infinie. Entremêlées, la constituaient toutes les choses qui seront, qui sont et qui furent. J'étais un fil dans cette trame totale, et Pedro de Alvarado, qui me tortura, en était un autre. Là résidaient les causes et les effets et il me suffisait de voir la Roue pour tout comprendre, sans fin (153)».

Cette Roue est la roue de l'univers faite de l'énergie cosmique qui est à la base de tout dynamisme. Avec l'eau et le feu, apparemment contradictoires, Tzinacan veut symboliser la vérité matérielle de la création et les deux sources complémentaires de toute vie.

Beaucoup de symboles suggérés par cette roue : monde cocyclique, circulaire, infini, cahier de l'éternité, facteur de l'espace-temps, l'eau et le feu avec leurs riches symboles. Dans ce cheminement nous guide (Brion, 2006) : «Le degré du rassemblement est ici supérieur, puisqu'au rassemblement spatial s'ajoute celui de tous les temps, de toutes les époques; l'on peut parler de " double rassemblement diégétique ».

L'eau est le symbole de la vie, la mère de toutes choses vivantes. Elle occupe soixante-quinze pour cent de la surface de la terre et soixante pour cent 
du corps humain. Concernant le symbolisme ${ }^{9}$ de l'eau, dans le livre de la Genèse $(1,1)$, l'esprit du Dieu s'est penché «au-dessus des eaux », dans la Bible, « l'eau qui lave les péchés du monde » (psaume 55). Dans la croyance chrétienne «nous naissons dans l'eau et ce n'est qu'en demeurant en elle que nous sommes sauvés » (Baptême I, 3). Dans le Coran, l'eau est l'essence de la vie «A partir de l'eau, nous avons produit toute chose vivante (XXI, 30), et que le trône du Dieu «était sur l'eau » (XI, 7).

Mais l'eau représente aussi la puissance écrasante des tsunamis dévastateurs, la purification, la clarté, la profondeur et le symbole de l'unité. Dans la culture chinoise, l'eau est Yin, et le feu est Yang, ils désignent respectivement la femme, "source intérieure : souple, patiente, capable de porter et de faire naître la vie. Dans le bouddhisme, la fleur de lotus ayant sa tige dans l'eau, symbolise "l'expérience" ${ }^{10}$. L'un des symboles qui révèle a quel degré Tzinacan a été opprimé en cherchant le salut et la liberté, «Pour l'Homme qui se mire et se cherche, l'eau devient un véritable écho visuel ${ }^{11}$.

Concernant le feu, selon Maryse Marsailly ${ }^{12}$, «il est lié à l'univers cosmique et solaire ». C'est aussi un symbolisme de la lumière et de la connaissance. Dans la mythologie Aztèque, Xiuhtecuhtli est dieu du feu. Pour eux, « la puissance du feu terrestre et souterrain permet l'union des contraires grâce à l'ascension et à la sublimation de l'eau en nuages. L'eau terrestre purifiée par le feu se transforme ainsi en eau céleste et divine ».

Ayant la forme circulaire, cette roue faite d'eau et de feu pourrait donc tourner à l'infini. C'est l'Univers cherché par Tzinacan, prisonnier en quête de liberté éternelle. La révélation de la roue expose les mots de Dieu devant ses yeux et le rend capable de voir le tracé de l'existence dans sa globalité : ce qui s'est passé et ce qui se produira, le sort de chaque vivant et de chaque objet inanimé. Il a pu discerner les tourmentés comme lui et les bourreaux comme Pedro de Alvarado. Il a saisi le principe de causalité en distinguant à fond entre le causant et le causé, les sens unidirectionnels ou bidirectionnels de cette causalité qui gouverne toute existence réelle.

Cette formule divine avec ses quatorze mots est la condensation symbolique de toutes les possibilités. L'investigation des récits de Borges révèle que l'usage des chiffres est associé généralement aux labyrinthes physiques et moraux où errent les hommes. Par exemple, la bibliothèque de Babel « se compose d'un nombre indéfini de galeries hexagonales » en notant que chaque hexagone possède six côtés. Dans La Demeure d'Astérion, Borges écrit :

\footnotetext{
${ }^{9}$ https://jesuschristsonenseignement.wordpress.com/tag/le-symbolisme-de-leau/

${ }^{10}$ Ibid

${ }^{11}$ Ibid.

${ }^{12} \mathrm{https}: / /$ blogostelle.blog/category/histoire-du-sacre/
} 
«Tu vas voir comme bifurque la cave. [...] Toutes les parties de celle-ci sont répétées plusieurs fois. Chaque endroit est un autre endroit. [...] Les mangeoires, les abreuvoirs, les cours, les puits sont quatorze [sont en nombre infini]. [...], les mers et les temples sont aussi quatorze [sont en nombre infini] [...] Tout est plusieurs fois, quatorze fois ».

Donc il est clair que le nombre quatorze chez Borges représente l'infini.

Se plonger dans la vision de différents êtres a permis à Tzinacan la connaissance de tous les chemins illimités qui mènent à la compréhension de l'écriture unique du Dieu, résumée en un seul mot couvrant toutes les informations infinies de cette existence. Chacun des quatorze mots contient l'information des autres mots, c'est-à-dire que l'information d'un mot est égale à l'information de tous les mots, sinon le mot deviendrait déficient, limité et cela contredirait la puissance infinie de la parole divine. Dans ce sens va Tzinacan avec sa réflexion :

«Avec le temps, la notion même d'une sentence divine me parut puérile et blasphématoire. Un dieu, pensai-je, ne doit dire qu'un seul mot et qui renferme la plénitude. Aucune parole articulée par lui ne peut être inférieure à l'univers ou moins complète que la somme du temps. Les pauvres mots ambitieux des hommes, tout, monde, univers, sont des ombres, des simulacres de ce vocable qui équivaut à un langage et à tout ce que peut contenir un langage (151)».

Ce raisonnement l'affirme Borges dans une de ses nouvelles où l'Aleph est une sorte de petite sphère contenant tous les savoirs de l'univers. C'est « l'un des points de l'espace qui contient tous les points (203)». Il ajoute qu' " aucun ne m'étonna autant que le fait que tous occupaient le même point (207) » et que « chaque chose (la glace du miroir par exemple) équivalait à une infinité de choses (208)» aussi que « le tout n'est pas plus grand que l'une des parties (212)».

Tzinacan qui a réussi à entamer cette communication profonde et illimitée avec le pouvoir divin est équivalant à tout homme qui suit sa démarche pour atteindre la vérité de l'univers, cet espace infini, dans lequel s'égalisent tous les choix «puisque devant l'infini, tous les choix se valent » comme indique Nicolas (1999). Tout dépend de l'homme et non de Dieu, du degré de sa clairvoyance, de son esprit ouvert à l'univers, de sa volonté d'acquérir le maximum du savoir universel dans le court chemin de sa vie, de sa faculté de se connaître. En effet, pour Borges, l'essentiel tend à valoriser sa faculté pensive afin de jouer le rôle d'un interlocuteur capable d'avoir accès à 
l'Écriture du Dieu, c'est-ce qu'indique Brion (2006) en disant «pour l'agnostique Borges donc, c'est finalement moins l'existence de Dieu qui est à remettre en cause que la capacité de l'homme à entrer en communication avec lui ; c'est la médiocrité humaine, plutôt que l'absence de Dieu, qui est pointée du doigt ».

Tzinacan est devenu le porte-parole de cette formule divine dont l'humanité a tellement besoin pour vivre en harmonie sur la Terre qui représente tout l'univers. Avec cette fameuse «formule de quatorze mots fortuits (qui paraissent fortuits) (154) », le mage est prêt à se venger de ses ennemis, de ses labyrinthes monstrueux, de l'obscurité de sa prison, de la férocité de ses bourreaux, de récupérer l'histoire brillante de son peuple, de retrouver sa place comme le Grand prêtre de la pyramide de Qaholom détruite par Pedro de Alvarado. Il lui suffirait de prononcer cette formule «pour anéantir cette prison de pierre, pour que le jour pénètre dans [sa] nuit, pour être jeune, pour être immortel, pour que le tigre déchire Alvarado, pour que le couteau sacré s'enfonce dans les poitrines espagnoles, pour reconstruire la pyramide, pour reconstituer l'empire (154)».

A cet égard, une grande question s'impose: Tzinacan, en tant qu'humain, est-il capable de prononcer ce mot divin ? Un mot qui fait parler tout l'univers avec ses infinies complexités ? À l'échelle humaine, est-ce que le fini peut-il contenir l'infini ? Forcément la réponse est non. Cette interrogation s'éliminerait seulement si Tzinacan pouvait s'unifier avec la divinité. C'est ce qui lui est arrivé, car son union avec Dieu lui a fait perdre son identité, et il n'est plus le mage prisonnier, mais il est devenu comme une goutte d'eau qui tombe dans la mer et perd sa propre identité pour se fondre dans celle de la mer qui seule peut prononcer ses propres mots. C'est pourquoi il est logique de constater que celui qui est uni avec le divin perd sa propre identité humaine qui est incapable de prononcer la parole éternelle de Dieu.

En effet, pour communiquer entre eux, les hommes sont condamnés à utiliser de divers langages régis par des limitations. Les noms des choses et des notions changent avec chaque langue qui est soumise à son univers physique et mental. Les lettres et les symboles ne peuvent pas transcender une réalité affranchie des facteurs spatio-temporels. Telle la lettre hébraïque "Aleph" qui «demeure toujours un signe abstrait qui ne fait pas vraiment partie du cosmos extérieur. Il ne se situe pas physiquement dans l'espace, ne possède pas de substance propre, ne s'investit jamais dans l'immanence. Nature et histoire lui demeurent étrangères par essence» comme affirme (Vigée, 2006).

Pour cette raison, l'être humain a besoin d'une langue non codée par des lettres pour atteindre la compréhension de l'Écriture du Dieu. Avec cette union avec le divin, Tzinacan n'est plus la même personne d'avant cette fusion et il en est conscient : 
« Mais je sais que je ne prononcerai jamais ces mots parce que je ne me souviens plus de Tzinacan (154)».

Qu'est donc devenu ce seul héritier des sages aztèques ? Est-il toujours héritier ? Certainement la réponse est négative car si elle était positive alors Tzinacan resterait lui-même et conserverait son propre nom, sa propre personnalité, et selon Claude Vigée «n'ayant plus conscience de sa propre identité, ne se souvenant plus de soi-même, la maîtrise des mots décisifs lui échappe également ». C'est ce qui est confirmé par la nouvelle " Que meure avec moi le mystère qui est écrit sur la peau des tigres (154)».

L'Écriture du Dieu ne s'est pas couronnée par la joie que Tzinacan a ressentie quand il a découvert la formule sacrée formée de quatorze mots, mais elle s'est conclue par une douleur ultime équivalente à celle du monde tout entier, en révélant les misères des hommes à travers tous les temps et tous les lieux.

Afin de retrouver la paix véritable, l'homme n'a qu'à se confondre avec le grand Tout. Avec cette fin, tous les hommes partagent cet oubli pour entrer dans un monde anonyme et infini sans ne se soucier ni du commencement du monde, ni de ceux qui les ont précédés ou viendront après eux. C'est pourquoi Tzinacan en tant que terrien se libère de tout affrontement inspiré par l'histoire réelle.

Grâce à sa persévérance, Tzinacan est parvenu à comprendre cette formule divine composée de quatorze mots, une sorte de quatorze notes musicales que l'Univers joue constamment. Son rêve mystique s'est transformé en une mélodie musicale magnifique et évocatrice qui résonne dans la solitude et le silence de tous ceux qui sont emprisonnés sans droit, qui aspirent à la liberté, à l'errance dans cet univers infini. Dans ce merveilleux sens va la mélodie ${ }^{13}$ intitulée "Le rêve de Tzinacan" composée par Fabien Waksman, (Kobekina, 2018) où le violoncelle d'Anastasia Kobekina incarne la voix du mage Tzinacan. Cette mélodie s'adresse à la profondeur de l'âme humaine afin de la libérer du labyrinthe des aléas de la vie. Cette précieuse pièce musicale «s'ouvre sur une mélodie circulaire s'appuyant sur une harmonie diatonique évoquant l'immuabilité de la situation du prisonnier. Le violoncelle, par ses élans lyriques successifs, amène la musique vers un premier sommet expressif figurant le souvenir de la sentence. Le discours se fait par la suite plus complexe et discontinu, la longue quête de l'écriture du Dieu étant parsemée de brusques moments de joie ou d'excitation comme d'instants de désespoir absolu ».

\footnotetext{
${ }^{13} \mathrm{https} / / /$ www.fabienwaksman.com/le-reve-de-tzinacan
} 


\section{Conclusion}

Dans cette nouvelle, Borges semble poussé par un désir d'absolu à la recherche d'une vérité pour tous les temps et tous les lieux réels ou fictifs. En faisant abstraction du réel et du présent, Tzinacan se projette vers l'avenir pour se libérer. Paradoxalement, les souvenirs viennent au secours du prisonnier pour le sauver des limites spatio-temporels. Ainsi, le temps vient au secours du prisonnier pour le sauver des limites de l'espace. C'est la victoire de l'immatériel sur le matériel. En effet, le combat du dernier prêtre de Qaholom est livré contre tout ce qui étouffe, tel le trou dans lequel il est jeté. Sa prison est celle de tous les humains prisonniers dans leurs propres labyrinthes naturels ou inventés. L'histoire de sa libération est l'exemple d'un affranchissement intellectuel qui pourrait amener l'être humain à délaisser sa faible Partie pour rejoindre le Tout sans parties.

Le parcours de ce mage incite l'homme à réfléchir, à apprendre, à découvrir en dialoguant avec la langue cosmique pour se libérer des particularités réductrices des langues humaines. En effet, l'une des caractéristiques de l'être humain est sa faculté d'écrire et de lire à travers des mots conventionnels afin de préserver ses patrimoines culturels et d'exprimer ses idées. L'association entre les signes et les valeurs propres à chaque communauté crée l'héritage écrit qui est à la base de toute sacralisation. Se trouver tributaire de ces limites, l'être s'emprisonne sans le savoir en se fermant aux valeurs des autres. A travers la mémoire, Tzinacan a su élargir ses connaissances au lieu de les réduire. En fortifiant cette faculté par la méditation et la réflexion, il a pu transcender les limites du physique, du temps et de l'espace en devenant un être à l'échelle de cet univers infini. Au lieu de périr dans sa finitude matérielle et intellectuelle, il a accédé à la formule philosophique et existentielle qui lui permet de se connecter à ses véritables origines en se confondant avec les fins cosmiques. L'emprisonnement physique n'est que la concrétisation palpable d'un enfermement psychique et intellectuel plus cuisant et plus profond. Par l'adhésion positive sans frontières à la mémoire collective, Tzinacan a choisi de se libérer dans l'infinité de l'univers. En effet, plus on sait, moins on est prisonnier de l'égoïsme.

La quête de Tzinacan est une recherche d'absolu pour vaincre le contingent. Et sa finitude n'est qu'une de ses formes : son corps affaibli et meurtri par la captivité marque le passage du temps. Ainsi, l'approche de la mort intensifie la sensation de la vie et les savoirs qui y sont attachés car son avancement initiatique ne s'est pas produit au début de son emprisonnement mais à la fin. Cela prouve qu'il y a un plus qui s'est produit dans sa douloureuse expérience : le sentiment intense de la finitude. A ce sujet a écrit (Dastur, 2007) : «Le se-savoir et le se-sentir mortel constituent le fondement de l'expérience que l'être humain a de lui-même. C'est donc cet étrange savoir, que chacun a avec certitude de sa propre fin et qui n'est semblable à 
aucun autre savoir du fait même de son irréductible dimension « affective », qui rend possible un discours non pas sur « la» mort, mais bien sur le rapport qu'entretient l'être pensant à sa propre mortalité. ». Dans le même sens va Lawrence Durrel (1957, p.200) « La présence de la mort rafraîchit toujours les expériences, c'est sa fonction : nous aider à méditer sur cette chose étrange qu'est le temps ». L'écriture divine recherchée par Tzinacan est l'essence de la pensée absolue car elle n'a ni début, ni fin. Et comme ses mots sont éternels et de dimension infinie, le mortel condamné et limité par nature est incapable de la prononcer comme l'affirme Lao Tseu «Un nom qui peut être prononcé n'est pas le Nom éternel » comme cité par (Watzlawick, 1985, p.354). Aussi, selon sa première proposition (La Pensée est un attribut de Dieu, autrement dit, Dieu est chose pensante), Spinoza ${ }^{14}$, explique qu'un étant/être pensant infini », un «étant qui peut penser une infinité de choses d'une infinité de manières ». Mais peut-être ce serait plus logique de dire que cette infinité de manières n'est que la seule manière divine. C'est pourquoi, quand Tzinacan a réussi à percevoir la vérité de "l'Écriture du Dieu", la vérité universelle, il a oublié son nom et tout ce qui est attaché à sa propre personne, y compris le désir de vengeance qui l'habitait. Sorti de son carcan particulier, il a abandonné sa finitude pour rejoindre l'éternité. Rêve ultime selon Borges pour dépasser l'écriture de l'homme prisonnière du temps et de l'espace. Par conséquent, l'humanité doit reconsidérer ses propres écritures, parce que le besoin de l'homme reste dans une écriture qui dépasse non seulement les frontières terrestres mais aussi les limites cosmiques. Borges veut que les hommes fassent de l'univers leur véritable demeure en préservant la planète Terre, qui est l'un des points infinis du cosmos. Cette demeure qui pourrait être périssable à son tour ne peut être magnifiée et débarrassée de ses maux que grâce à l'intelligence humaine qui se perpétue exclusivement à travers la pensée. Leur ambition de retrouver la sentence divine qui pourrait les sauver ne peut être possible qu'à force d'une évolution intrinsèque à leurs esprits qui les délivre de leurs diverses prisons visibles et invisibles.

Tzinacan s'est libéré des contraintes de son identité humaine limitée en la remplaçant par une identité cosmique qui ne connaît qu'elle-même et elle ne s'explique que d'elle-même. Cela ne se réalise qu'à travers l'union divine. C'est pourquoi, Tzinacan s'est séparé complètement de son soi mortel afin de se dissoudre dans l'éternité. Après avoir atteint cette extase divine, les paroles de Tzinacan ne ressemblent plus aux mots humains et par conséquent, il lui est donc devenu impossible de les prononcer. Ainsi, l'écriture divine est prononcée par l'univers avec tous ses phénomènes et toute son infinité dans une harmonie éternelle entre les normes et les grandeurs cosmiques. La formule divine est cette union indescriptible entre les mots et les choses, et

\footnotetext{
${ }^{14} \mathrm{https}: / /$ spinoza.fr/lecture-des-propositions-i-a-vii-du-de-mente/\#.
} 
donc il est impossible de prononcer l'écriture divine par des mots à l'échelle humaine parce que «la langue, comme performance de tout langage, n'est ni réactionnaire, ni progressiste; elle est tout simplement: fasciste; car le fascisme, ce n'est pas d'empêcher de dire, c'est d'obliger à dire » comme enseigne (Barthes, 1977, p.14). Dans cette optique, Borges voit que le seul moyen pour l'homme d'échapper à ces " pauvres mots ambitieux des hommes (151) », à cet égarement perpétuel dans les particularités est d'accepter d'être un constituant parmi d'autres dans la roue suprême constituée par le cosmos.

Ainsi, Tzinacan s'est servi de la mémoire pour se ressaisir et s'appuyer sur un socle pour s'élancer dans sa quête d'absolu, mais quand les données de cette mémoire deviennent une entrave à sa progression il les élimine volontiers. Et comme le dit (Borges, 1970) «L'oubli et la mémoire sont également inventifs ». En suivant le chemin de sa mémoire il a pu conquérir l'amnésie bienfaisante de la fin. Ce n'est pas l'anéantissement, c'est l'anonymat qui est une renaissance véritable. C'est l'oubli victorieux. En effet, Tzinacan accède à l'état de l'anonymat comme destination ultime, incontournable et naturelle de tout être. Il se libère ainsi de toutes les définitions temporelles : gloire, triomphe et puissance n'ont plus les mêmes significations. La dignité de sa défaite se transforme en une victoire éclatante sur les dimensions spatiales et temporelles autrement sur la finitude terrestre. Les expériences anciennes doivent être dépassées pour atteindre des stades supérieurs et les affres des labyrinthes vécues par Tzinacan sont les marques d'une existence souffrante et enchaînée par les signes, les symboles, les détails labyrinthiques qui ne peuvent aboutir qu'à de nouveaux supplices toujours renaissants. Le passé et le présent doivent servir un cheminement initiatique profond semé d'épreuves symbolisées par les images de labyrinthes que le vainqueur a enrayées en définitif.

L'oubli ultime auquel accède le héros avec bonheur est un état extatique réservé aux initiés. Cette sublimation hors mesure ne peut être révélée que dans des expériences mystiques. Dans ce sens va (Spychiger, 2014) dans la conclusion de sa thèse en soulignant que l'attitude générale de Borges consiste à placer «ses personnages, dans des états de conscience particuliers (l'extase, la méditation, la contemplation, le rêve, le dédoublement...), afin de les faire accéder à un au-delà de la représentation, ce qui les conduit à des révélations, jusque-là demeurées secrètes et inaccessibles».

\section{References:}

1. Barthes, R. (1977). Leçon. Paris : Éditions du Seuil.

2. Bergson, H. (1896, 2011). Matière et mémoire : Essai sur la relation du corps à l'esprit. Edition numérique : Pierre Hidalgo. La Gaya Scienza.

3. Borges, J. (1969). Éloge de l'Ombre. Cambridge. 
4. Borges, J. (1970). Le Rapport de Brodie, traduit de l'espagnol par Françoise-Marie Rosset 1972. Paris: Gallimard.

5. Borges, J. L. (1953). Labyrinthes. Trad. de l'espagnol (Argentine) par Roger Caillois. Paris: Gallimard.

6. Borges, J. L. (1953). L'ALEPH; Traduit de l'espagnol par Roger Gaillois et René L.-F. Durand (1967). Paris: Gallimard.

7. Borges, J. L. (1975). Le livre de sable. Traduction revue par JeanPierre Bernes. Paris: Gallimard, 1978.

8. Borges, J.-L. (1936, 1971). Historia de la eternidad (Histoire de l'éternité), essai "El tiempo circular" (Le temps circulaire). trad. de l'espagnol par Roger Caillois et Laure Guille, Paris, U.G.E. Paris: UGE.

9. Brion, C. (2006). L'ÉCRITURE BORGÉSIENNE DU MONDE : UNE CONJURATION FANTASTIQUE DU DÉSORDRE ? Revue de littérature comparée, 4(320), 491-496.

doi:https://www.cairn.info/revue-de-litterature-comparee-2006-4page-491.htm

10. Brotherston, G. (2011). Aleph and Borges. Variaciones Borges (31), 233-238.

11. Chaumillon, É. (2016). L'exploitation des sables et granulats marins : une menace pour les littoraux. L'Institut océanographique. www.institut-ocean.org.

12. Civitarese, G. (2015). Rêves De Rêves. Traduit de l'italien par Laura Cecotti-Stievenard. Société de Psychanalyse Freudienne | "Les Lettres de la $S P F$ », 1(33), 95-111. doi:https://www.cairn.info/revueles-lettres-de-la-spf-2015-1-page-95.htm

13. Dastur, F. (2007). La mort: Essai sur la finitude. Collection : Épiméthée (éd. Presses Universitaires de France).

14. Fontcuberta, J., Borges, J. L., \& Bleton, C. (2000). BORGES ET MOI dans « La pensée de midi ». 2(2), 52-59.

doi:https://www.cairn.info/revue-la-pensee-de-midi-2000-2-page52.htm

15. Girgois, H. (2015). L'Occulte chez les aborigènes de l'Amérique du Sud. Date de l'édition originale : 1897. LIGARAND.

16. Kobekina, A. (2018). Le rêve de Tzinacán pour Violoncelle et orchestre à cordes. Waksman: compositeur, Anastasia Kobekina tient la partie de violoncelle solo. doi:https://www.fabienwaksman.com/lereve-de-tzinaca

17. Lavarde, A. (1999). La sphère, symbole du mouvement. Communication \& Langages (119), 55-65.

18. Mathieu-Colas, M. (2017). Dictionnaire des noms de divinités. Paris. doi:https://halshs.archives-ouvertes.fr/halshs-00154061/document 
19. Mottet, P. (2010). Les jeux de la réécriture : L'Aleph de Borges ou la re-création du monde. Québec français (159), 42-45.

20. Nicolas, L. (1999). Borges et l'infini. Variaciones Borges (7), 88-146.

21. Shroukh, S. (2020). Livres de Chilam Balam (p. 1027-1039): ENCYCLOPÉDIE DES HISTORIOGRAPHIES : AFRIQUES, AMÉRIQUES, ASIES. (N. Kouamé, É. . P.Meyer, \& A. Viguier, Éd.) Paris: Presses de l'Inalco.

22. Spychiger, D. (2014). Borges, le poète, ou le miroir universel du monde. Université de Pau et des Pays de l'Adour.Laboratoire llcaa. Doctorat Hispanophone.

23. Vigée, C. (2006). Borges devant La Kabbale Juive de l'écriture du dieu au silence de l'Aleph. Revue de littérature comparée, 4(320), 397-413. doi:https://www.cairn.info/revue-de-litterature-comparee-2006-4page-397.htm

24. Watzlawick, P. (1985). L'invention de la réalité: contributions au constructivisme. Traduit de l'allemand par Anne-Lise Hacker. Paris: Éditions du Seuil,1988.

25. Zinn, H. (2009). A People's History of the United States: 1492 to Present. doi:https://jugurtha.noblogs.org/files/2018/05/HowardZinnHistoirepopulairedesEtats-Unis.pdf

26. https://spinoza.fr/lecture-des-propositions-i-a-vii-du-de-mente/\#.

27. https://www.fabienwaksman.com/le-reve-de-tzinacan

28. https://blogostelle.blog/category/histoire-du-sacre/

29. https://jesuschristsonenseignement.wordpress.com/tag/lesymbolisme-de-leau/

30. https://www.museedelhistoire.ca/cmc/exhibitions/civil/maya/mmj01f ra.html\#.

31. https://www.jepense.org/symbolisme-du-cercle/

32. https://bruxelles-panthere.thefreecat.org/,

33. https://www.matierevolution.fr/spip.php,

34. https://jugurtha.noblogs.org/files/2018/05/HowardZinnHistoirepopulairedesEtats-Unis.pdf 